\title{
VISCOELASTIC PULSE PROPAGATION AND STABLE PROBABILITY DISTRIBUTIONS*
}

BY

ANDREAS KREIS AND A. C. PIPKIN

Brown University

1. Introduction. The purpose of the present note is to point out an interesting connection between viscoelastic pulse propagation and the theory of infinitely divisible and stable probability distributions. Velocity pulses in viscoelastic materials have the same shapes as infinitely divisible probability densities, and when the viscoelastic material response is of the kind that is typical for stiff polymeric materials, the velocity pulse takes the shape of a stable probability density.

In Sec. 2 we explain the equations and boundary conditions governing one-dimensional wave propagation in a semi-infinite body of linearly viscoelastic material, and obtain the Laplace transform of the velocity $V(y, t)$ for the case in which the input velocity is a Dirac delta, $V(0, t)=\delta(t)$. The viscoelastic material is assumed to have a creep compliance whose slope is completely monotone. We consider in particular the case of a power-law compliance, a model that is a good approximation to the behavior of some polymeric materials.

In Sec. 3 we explain the definitions of infinitely divisible and stable probability densities for positive random variables, and show the connection with viscoelastic pulse shapes. At any fixed station $y$, the velocity $V$ as a function of time is an infinitely divisible probability density. When the compliance has the power-law form, the pulse shape (in time, at a given station) is one of the stable densities.

The remainder of the paper concerns the pulse shape in a power-law material. In the limiting cases of perfectly elastic and perfectly viscous behavior the Laplace transform can be inverted explicitly, and in the general case asymptotic approximations valid for small and large times can be obtained. We show this in Sec. 4.

In the case of a perfectly elastic material, the pulse response function is a Dirac delta. For a power-law material whose response is very nearly elastic, the pulse response becomes a narrow, sharply peaked function. Kolsky [1] observed experimentally that the

*Received July 18, 1985. 
shape of this function is nearly independent of the power-law parameter when the response is close to elastic, and calculated this universal pulse shape by a Fourier series method. In Sec. 5 we explain a different approach to the calculation of the pulse shape, based on an approximation to the inversion integral, and in Sec. 6 we show that the approximate inversion integral can be reduced to a real integral over a finite interval by integrating along a steepest descent path. This integral can be evaluated numerically. In Sec. 7 we discuss the numerical results.

Except for the results in Secs. 6 and 7, everything in the present paper has been published previously. The results on probability are from the book by Feller [2], and those on viscoelasticity are from the book by Pipkin [3].

2. Viscoelastic pulse propagation. The momentum equation and stress-strain relation for one-dimensional shear wave propagation in a homogeneous, linearly viscoelastic material are, respectively,

$$
\rho v_{t}=\sigma_{y}
$$

and

$$
u_{y}=J * \sigma_{t},
$$

where

$$
v=u_{t} .
$$

Here $\rho$ is a positive constant, the density; $u(y, t)$ is the particle displacement and $v(y, t)$, its time derivative, is the particle velocity; and $\sigma(y, t)$ is the shearing stress. $J(t)$ is a specified function called the creep compliance. It is zero for $t<0$. For $t \geqslant 0$ we assume that $J$ is nonnegative and that its derivative $J^{\prime}$ is completely monotone, i.e., its derivatives alternate in sign:

$$
J>0, J^{\prime}>0, J^{\prime \prime}<0, J^{\prime \prime \prime}>0, \ldots \quad(t>0) .
$$

We also admit as limiting cases the case of an elastic material, for which $J$ is a positive constant, and a viscous fluid, for which $J=t / \eta$, where $\eta$ is a positive constant. For these materials the relations (2.4) are not satisfied as strict inequalities. The assumption that $J^{\prime}$ is completely monotone is not known to be contradicted by experimental evidence for any material.

The star in (2.2) denotes convolution with respect to the variable $t$ :

$$
f * g=\int_{-\infty}^{t} f\left(t-t^{\prime}\right) g\left(t^{\prime}\right) d t^{\prime}
$$

We consider a half-space $y>0$ that is initially quiet, so that

$$
\sigma=u=v=0 \quad(t \leqslant 0, y>0) .
$$

At time zero the boundary $y=0$ is subjected to a unit step displacement, or thus a Dirac delta velocity pulse:

$$
v(0, t)=\delta(t) \quad(-\infty<t<\infty)
$$


The velocity $V(y, t)$ that satisfies the preceding conditions is the basic pulse response function for the material. We find that its Laplace transform is

$$
\bar{V}(y, s)=\exp \left[-y s(\rho s \bar{J})^{1 / 2}\right],
$$

where $\bar{J}$ is the transform of $J$. In the particular case when $J$ is proportional to a power of $t$, so that

$$
J(t)=J_{0} t^{2 \epsilon} /(2 \epsilon) ! \quad(0 \leqslant 2 \epsilon \leqslant 1),
$$

the transform $\bar{J}$ is also a power,

$$
s \bar{J}=J_{0} s^{-2 \epsilon} .
$$

In this case the parameters $\rho$ and $y$ in the transform of $V$ can be suppressed by scaling. Thus, if $K(t ; \epsilon)$ is the function whose transform is

$$
\bar{K}(s ; \epsilon)=\exp \left(-s^{1-\epsilon}\right),
$$

then

$$
V(y, t)=\left(y_{1} / y\right)^{p} K\left[t\left(y_{1} / y\right)^{p} ; \epsilon\right]
$$

where

$$
y_{1}=\left(\rho J_{0}\right)^{-1 / 2}, \quad p=1 /(1-\epsilon) .
$$

The function $K$ represents the pulse shape as a function of time at the station $y=y_{1}$, and we see that the shape at other values of $y$ differs from this only by scale factors.

3. Stable probability distributions. Let $f(t)$ be the probability density of a positive random variable. The density $f$ is infinitely divisible if for each positive integer $n$ there is a density $f_{n}$ such that

$$
f=f_{n} * f_{n} * \cdots * f_{n} \quad(n \text { factors }),
$$

i.e., $f$ can be represented as the density of a sum of $n$ identically distributed positive random variables. For this to be true, it is necessary and sufficient that the Laplace transform of $f$ have the form ([2], XIII.7)

$$
\bar{f}(s)=\exp (-F(s))
$$

with $F(0)=0$ and $F^{\prime}(s)$ a completely monotone function for real $s>0$. An infinitely divisible distribution can be imbedded in a family numbered with a parameter $y$, such that

$$
f(x+y)=f(x) * f(y) .
$$

From its transform (2.9), we see that the pulse response function $V(y, t)$ forms such a family of infinitely divisible densities provided that $s(s \bar{J})^{1 / 2}$ has a completely monotone derivative. It can be verified ([3], III.9) that this is true if $J^{\prime}$ is completely monotone for $t>0$. Thus, the pulse shape recorded on an oscilloscope in a viscoelastic pulse propagation experiment is an infinitely divisible probability density.

A family of infinitely divisible distributions, satisfying (3.3), is said to be stable if the various members of the family are all the same after appropriate rescaling with respect to the parameter $y$. The family of densities with transforms of the form $\exp \left(-y s^{1-\epsilon}\right)$ 
$(0<\epsilon<1)$ is stable ([2], XIII.6). Comparison with (2.12) shows that the pulse shape $K(t$; $\epsilon)$ in a power-law solid is one of these stable densities.

4. Special values of the pulse response function. The function $K(t ; \epsilon)$ can be evaluated explicitly in the limiting cases of perfectly elastic and perfectly viscous behavior. For $\epsilon=0$ the viscoelastic material with compliance (2.10) is perfectly elastic, and the input pulse propagates with no dispersion or attenuation:

$$
K(t ; 0)=\delta(t-1) .
$$

For $2 \epsilon=1$ the material is a viscous fluid, and the pulse response has the form

$$
K(t ; 1 / 2)=(1 / 2 \sqrt{\pi}) t^{-3 / 2} \exp (-1 / 4 t) .
$$

By using the power-series expansion of the transform (2.12), a series valid asymptotically as $t$ approaches infinity can be obtained ([2], XVII.6):

$$
K(t ; \epsilon) \sim \frac{1}{\pi t} \sum_{1}^{\infty} \frac{[n(1-\epsilon)] !}{n !} \sin (n \pi \epsilon) t^{-n(1-\epsilon)} \quad(t \rightarrow \infty) .
$$

By using a saddle-point approximation to the inversion integral for (2.12), an approximation valid asymptotically as $t$ approaches zero can be obtained ([3], IV.2.c):

$$
K(t ; \epsilon) \sim \frac{1}{\sqrt{2 \pi}} \frac{1}{\epsilon t} \sqrt{1-\epsilon} \sqrt{X} \exp (-X) \quad(t \rightarrow 0),
$$

where

$$
X=\epsilon[(1-\epsilon) / t]^{(1 / \epsilon)-1} .
$$

Comparison with (4.2) shows that (4.4) is exact when $\epsilon=1 / 2$. For other values of $\epsilon,(4.4)$ disagrees with (4.3) when $t$ is large ( $X$ small), although it correctly indicates that $K$ approaches zero again when $t$ is large.

5. Nearly elastic materials. In the limit as the parameter $\epsilon$ approaches zero we can anticipate from $(4.1)$ that $K(t ; \epsilon)$ will be very sharply peaked near $t=1$, with a long tail asymptotic to $\epsilon / t^{2-\epsilon}$ for $t$ large [from (4.3)]. The main pulse shape, but not the tail, can be computed approximately by using a variant of the saddle-point method ([3], IV.2.c).

The inversion integral for the transform (2.12) is

$$
K(t ; \epsilon)=(2 \pi i)^{-1} \int \exp \left(s t-s^{1-\epsilon}\right) d s .
$$

The exponent is stationary at the point $s_{0}$ defined by

$$
t=(1-\epsilon) s_{0}^{-\epsilon} ; \quad s_{0}=X / \epsilon t,
$$

where $X$ is defined in (4.5). Instead of expanding the exponent in powers of $s-s_{0}$ as is normally done in the saddle-point method, it is more accurate when $\epsilon$ is small to expand $s^{-\epsilon}$ in powers of $\epsilon \ln \left(s / s_{0}\right)$. With $z=s / s_{0}$, the exponent becomes

$$
s t-s^{1-\epsilon}=X z\left[\ln z-1-(\epsilon / 2)(\ln z)^{2}+O\left(\epsilon^{2}\right)\right] .
$$


Then in the limit as $\epsilon$ approaches zero with $X$ fixed, $K$ is given asymptotically by

$$
K(t ; \epsilon) \sim(1 / \epsilon t) I(X) \quad(\epsilon \rightarrow 0)
$$

where

$$
I(X)=(X / 2 \pi i) \int \exp [X(z \ln z-z)] d z .
$$

The saddle-point approximation to (5.5) for large $X$ yields

$$
I(X) \sim(X / 2 \pi)^{1 / 2} \exp (-X) \quad(X \rightarrow \infty),
$$

in agreement with (4.4) for small $\epsilon$. However, (5.4) is valid for small $X$ as well, if $I(X)$ is evaluated exactly.

Higher-order approximations to $K$ can be obtained by standard methods. Several terms of the resulting expansion can be combined to yield

$$
K(t ; \epsilon)=(1 / \epsilon t)\left[(1-\epsilon)^{1 / 2} I(X)+O\left(\epsilon^{2} / X\right)\right] .
$$

From this form we see that if $I(X)$ is evaluated exactly, the resulting approximation is numerically valid so long as $X$ is large in comparison to $\epsilon^{2}$. As we show in Sec. 7, the main pulse response is entirely inside this region when $\epsilon$ is small. The final die-out for $t \rightarrow \infty$, given by (4.3), is not given by (5.7).

6. Steepest descent evaluation. The function $I(X)$ defined by (5.5) can be expressed as a real proper integral by integrating along a steepest descent path through the saddle point $z=1$. The steepest descent path is defined by

$$
\operatorname{Im}(z \ln z-z)=0 .
$$

With $z=r e^{i \theta}, r$ is given as a function of $\theta$ along this path by

$$
r(\theta)=\exp [1-\theta / \tan \theta] \text {. }
$$

On this path,

$$
z \ln z-z=-r \theta / \sin \theta=-f(\theta) \quad(\text { say })
$$

and

$$
d z=(\operatorname{Re} d z)+i f(\theta) d \theta .
$$

The integral in (5.5) is thus converted into an integral with respect to $\theta$ over the interval $[-\pi, \pi]$. The real part of $d z$ furnishes an odd integrand that integrates to zero, and the remaining integrand is even. Then

$$
I(X)=(X / \pi) \int_{0}^{\pi} \exp [-X f(\theta)] f(\theta) d \theta .
$$

The integral (6.5) can be evaluated numerically. The function $I(X)$ obtained by doing this is shown in Fig. 1, where it is compared with the saddle-point approximation (5.6). It is seen that the saddle-point approximation is still quite good even at relatively low values of $X$. The maximum of $I(X)$ occurs near $X=1 / 2$, the place where the approximation (5.6) is maximum, and the exact value of $I(X)$ there is only about $6 \%$ higher than the approximate value. 


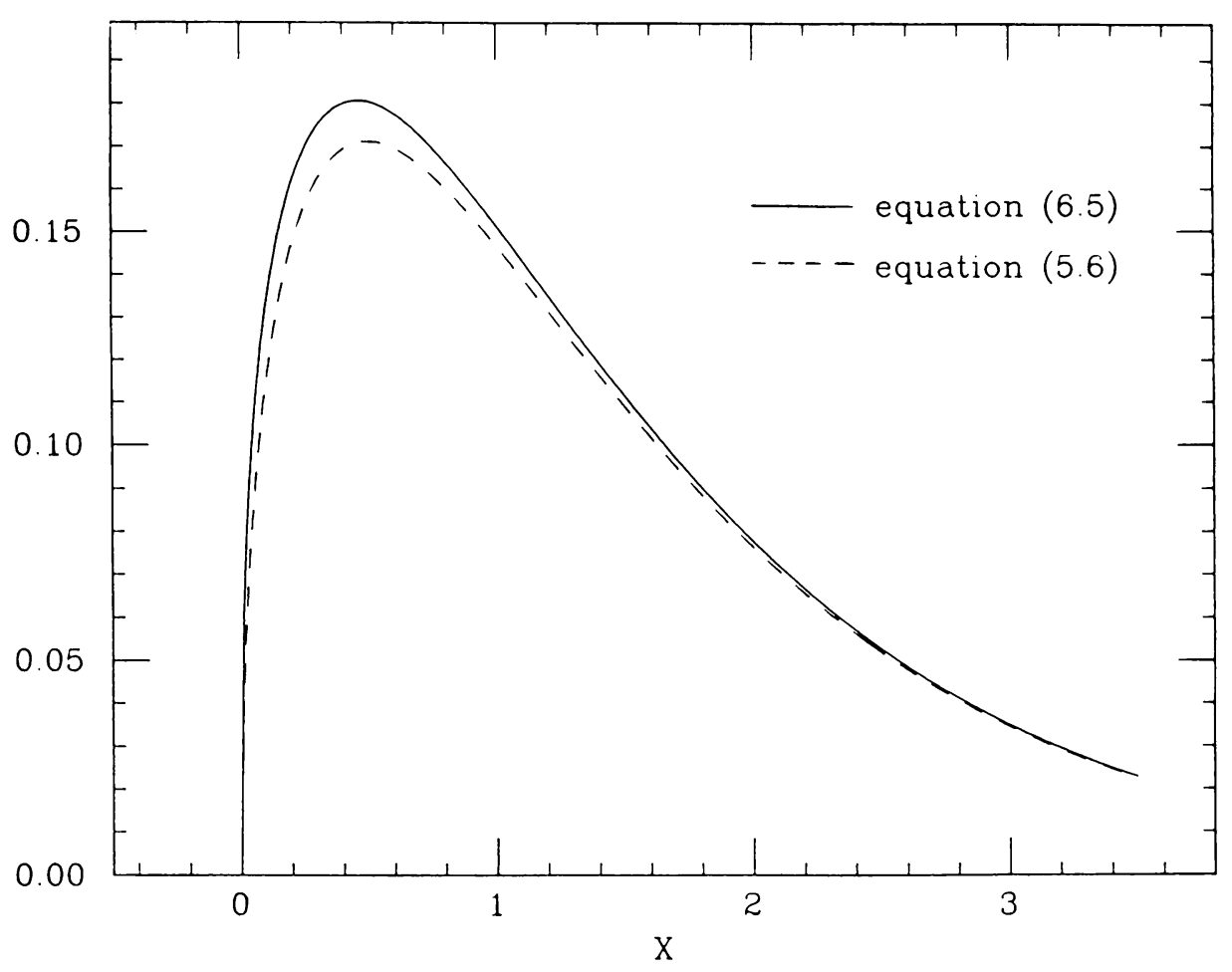

FIG. 1. The function $I(X)$ (upper curve) and the saddle-point approximation to it (lower curve).

7. Kolsky's universal pulse shape. In pulse propagation experiments on polyethylene, polystyrene, and poly(methyl methacrylate), Kolsky [1] observed that the pulse shapes were independent of the distance of the observation station from the pulse source, merely decreasing in amplitude and broadening in width as the distance from the source increases. He also observed that although the pulse shapes were quite unsymmetrical, the pulse shapes for different materials could be brought into good congruence by scaling them appropriately with the loss angle $\delta$ (a material parameter) as a scale factor. The pulse widths were proportional to $\delta$, and their amplitudes were inversely proportional to $\delta$. Kolsky explained this theoretically as a consequence of the fact that the loss angle is small and nearly constant over a broad range of frequencies for each material considered. By using this assumption, he computed a universal pulse shape by a Fourier series method (a fast Fourier transform).

The loss angle is exactly constant for materials whose compliance has the power-law form (2.10), being $\delta=\epsilon \pi$. In the present method of calculation, the universal pulse shape should be the function $\epsilon K$, expressed as a function of $\left(t-t_{m}\right) / \epsilon$, where $t_{m}$ is the time at which $K$ reaches its maximum. From (5.4) we see that better agreement among various small values of $\epsilon$ can be obtained by comparing values of $\epsilon t_{m} K$. Since $t$ is given in terms 
of $X$ [from (4.5)] by

$$
t=(1-\epsilon)(\epsilon / X)^{\epsilon /(1-\epsilon)},
$$

and $I(X)$ has its maximum near $X=1 / 2, t_{m}$ is approximately $(2 \epsilon)^{\epsilon}$ when $\epsilon$ is very small. Thus $t_{m}=1$ in the limit $\epsilon \rightarrow 0$, but $t_{m}$ approaches this limiting value only slowly. From (4.5) we also see that in the vicinity of the peak, where $X=X_{m}$,

$$
X-X_{m}=(1-\epsilon)\left(X_{m} / t_{m}\right)\left(t-t_{m}\right) / \epsilon+\cdots,
$$

and thus when the pulse shape (5.4) is expressed in terms of $t$, the pulse width is proportional to $\epsilon$ in the lowest order of approximation.

In Fig. 2 we show graphs of $\epsilon t_{m} K$, or rather $\left(t_{m} / t\right) I(X)$, versus the scaled time variable defined by the right-hand member of (7.2), in which we use the exact expression (7.1) for $t(X)$. Graphs for the values $\epsilon=0.1,0.01$, and 0.001 are shown. The curves for the two smaller values of $\epsilon$ cannot be distinguished on the scale shown here, although of course they are not exactly equal. The factor $(1-\epsilon)^{1 / 2}$ in the improved approximation (5.7) has been omitted here.

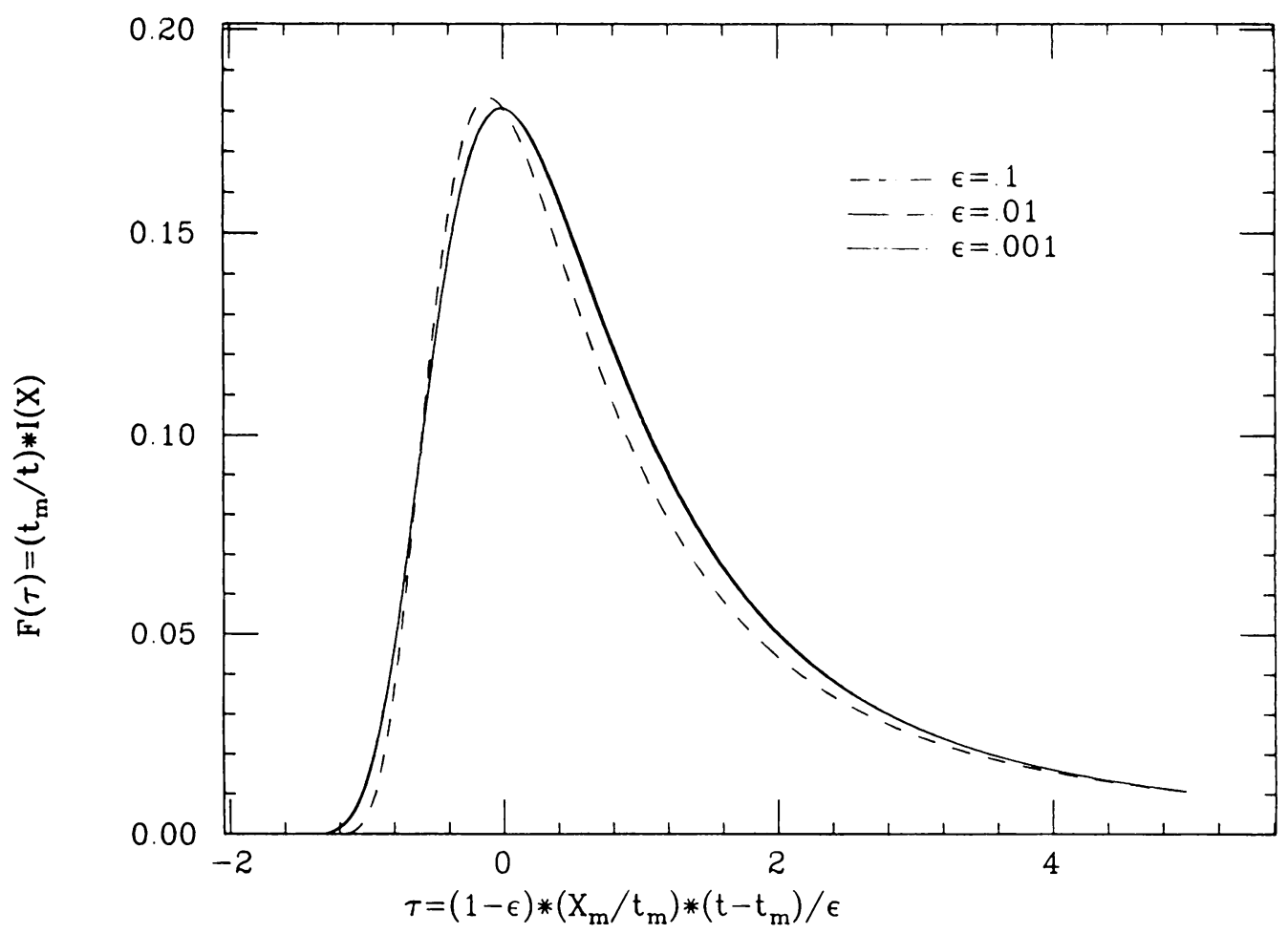

FIG. 2. The universal pulse shape for nearly elastic materials. 
Acknowledgment. The work described in this paper was carried out under grant DMS-8403196 from the National Science Foundation and a fellowship from the Swiss National Foundation. We gratefully acknowledge this support.

\section{REFERENCES}

[1] H. Kolsky, The propagation of stress pulses in viscoelastic solids, Philos. Mag., Ser. 8, 2, 693-710 (1956)

[2] W. Feller, An introduction to probability theory and its applications, vol. II, Wiley, New York (1966)

[3] A. C. Pipkin, Lectures on viscoelasticity theory, 2nd ed., Springer-Verlag, New York (1986)

\section{DYNAMICS: THE GEOMETRY OF BEHAVIOR}

Abraham and Shaw

A pictorial introduction to non-linear dynamics: attractors, basins, separatrices, chaos and bifurcations.

\section{Part I: Periodic Behavior} 342 illustrations, 4-color, 220 pages, $\$ 34$

Part II: Chaotic Behavior 170 illustrations, 2-color, 137 pages, $\$ 28$

Part III: Global Behavior 136 illustrations, 2-color, 123 pages, $\$ 28$

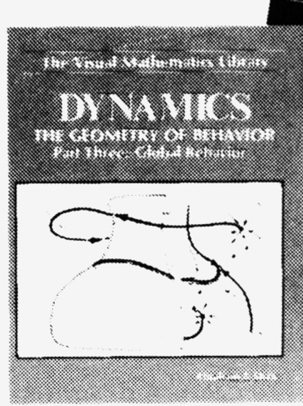

Add \$2/book in Canada, \$10/book in all other countries.

ONE-STOP SHOPPING FOR ALL YOUR DYNAMICS NEEDS: Abraham, Tufte, Fischer and Hayashi books NOW IN STOCK. IBM and Apple disks and films NOW IN STOCK. Available direct from the publisher: AERIAL PRESS, INC., P.O. Box 1360, Santa Cruz, CA 95061. (408) 425-8619. 